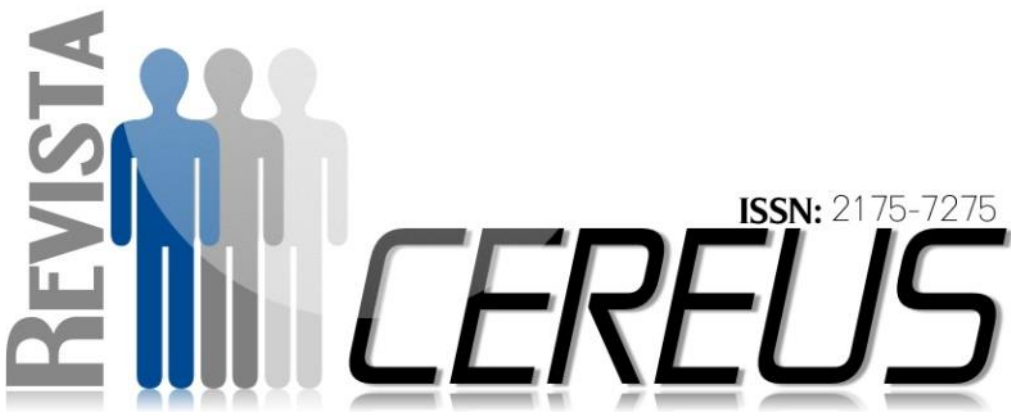

\section{CRITÉRIOS DE AVALIAÇÃo dA ATENÇÃo PARA PESSOAS VIVENDO COM HIV/Aids}

RESUMO

ROMÃO, Tiago Carnevalle ${ }^{1}$

MARSON, Poliana Guerino ${ }^{2}$

SANTOS, Leidiene Ferreira ${ }^{3}$ EVANGELISTA, Danielle Rosa ${ }^{3}$ SILVA, Tiago Barreto de Castro ${ }^{4}$

Objetivo: analisar as evidências relacionadas aos critérios de avaliação da atenção em PVHA presentes na literatura científica. Método: Trata-se de uma revisão integrativa, tendo a prática baseada em evidências como referencial metodológico. Para a seleção dos estudos foram aplicados descritores junto às bases de dados PUBMED, LILACS e Web of Science resultando em 474 estudos. Após aplicação dos critérios de inclusão e exclusão, foi obtida uma amostra final de 12 artigos lidos na íntegra. Resultados: os estudos evidenciaram a assistência em pessoas vivendo com

\footnotetext{
${ }^{1}$ Mestre. Professor D I - 1 do Instituto Federal de Educação, Ciência e Tecnologia do Tocantins. e-mail: tiago.romao@ifto.edu.br

${ }^{2}$ Doutora. Professora adjunta 2 do Curso de Medicina da Universidade Federal do Tocantins.

${ }^{3}$ Doutora. Professora adjunta 1 do Curso de Enfermagem da Universidade Federal do Tocantins.

${ }^{4}$ Doutor. Professor adjunto 1 do Curso de Enfermagem da Universidade Federal do Tocantins.
} 
HIV/Aids (PVHA) focada no método convencional de terapia antirretroviral; disponibilidade de recursos humanos, equipamentos e de estrutura física, estratégia de aconselhamento e testagem, estratégia de prevenção da transmissão vertical do HIV, acesso e comunicação usuárioserviço de saúde. Conclusão: Os critérios de avaliação presentes na dimensão organizacional e da disponibilidade de recursos apresentaram relevância quanto as boas práticas assistenciais como redução da mortalidade, melhora da qualidade de vida, asseguram a acessibilidade, promovem cidadania e bem-estar social, entretanto, ainda há a necessidade de estudos que ampliem os parâmetros assistenciais às PVHA.

Palavras chave: HIV/Aids. Critérios de avaliação. Serviços de saúde.

\section{ATTENTION EVALUATION CRITERIA FOR PEOPLE LIVING WITH HIV/AIDS}

\section{ABSTRACT}

Objective: To analyze the evidence related to the evaluation criteria of attention in PLWHA present in the scientific literature. Method: This is an integrative review, with evidence-based practice as a methodological framework. For the selection of the studies, descriptors were applied to the PUBMED, LILACS and Web of Science databases resulting in 474 studies. After applying the inclusion and exclusion criteria, a final sample of 12 articles read in full was obtained. Results: the studies showed assistance in people living with HIV / AIDS (PLHA) focused on the conventional method of antiretroviral therapy; availability of human resources, equipment and physical structure, counseling and testing strategy, prevention strategy for vertical HIV transmission, access and communication between users and health services. 
Conclusion: The evaluation criteria present in the organizational dimension and the availability of resources have shown relevance in terms of good assistance practices, such as mortality reduction, quality of life improvement, accessibility, citizenship and social welfare, however, there is still a need for studies that increase the assistance parameters to PLHA.

Keywords: HIV/AIDS. Evaluation criteria. Health services. 


\section{INTRODUÇÃO}

Nos últimos 30 anos a aids trouxe severos danos a diversas famílias e comunidades pelo mundo constituindo uma das grandes preocupações de saúde pública. O relatório global do Programa Conjunto das Nações Unidas sobre HIV/Aids (UNAIDS) estimou que 36,7 milhões de pessoas vivem com o HIV no mundo (UNAIDS, 2016a). Ademais, estima-se, globalmente, que sejam infectados, diariamente, $\quad 5.700$ indivíduos (UNAIDS, 2016). A doença é a $5^{\text {a }}$ causa de morte entre os adultos e a principal entre mulheres da faixa etária de 15 a 49 anos (UNAIDS, 2012).

A pandemia do HIV/aids, caracterizada como sendo um problema de saúde pública mais amplo, trouxe danos ao desenvolvimento econômico e social. Além dos custos de prevenção e tratamento, que são bastante elevados, são afetados diretamente os fatores de produção de uma nação: riqueza, trabalho e capital intelectual, impactando negativamente o crescimento econômico do país (WORLD HEALTH ORGANIZATION, 2016; GODOY; SOUSA; SILVA, 2013).

Para o sucesso de programas de saúde dirigidos às pessoas portadoras de doenças crônicos, tornase necessário a práticas que sejam determinantes na qualidade assistencial (MELCHIOR et al., 2006). A prática assistencial bem dirigida resulta em impactos positivos na qualidade de vida, nas reduções da mortalidade e da epidemia do HIV (BRASIL, 2016; MELCHIOR et al, 2006).

\section{$1.1 \quad-\quad$ REFERENCIAL}

\section{TEÓRICO}

Avaliar um programa ou estratégia significa, muitas vezes, realizar a apreciação valorativa de uma determinada intervenção que se baseia em procedimentos apoiados no método científico para justificar o mérito de algo. A pesquisa avaliativa não possui método e técnicas próprias, utiliza-se de métodos da pesquisa social (AGUILAR; ANDER-EGG, 1994). Para Castanheira (2002), a pesquisa avaliativa na saúde consiste em utilizar do método científico para julgar o valor de uma intervenção construída pela necessidade social.

Ao adotarmos a "qualidade" como parâmetro avaliativo de uma estratégia ou programa de saúde, nos 
valemos de um conceito originado de uma das mais conhecidas vertentes da avaliação em saúde. Adevis Donabedian, um dos pioneiros do conceito de qualidade em saúde nos anos de 1960, é autor das dimensões baseadas na estrutura-processoresultado, que devem ser avaliadas na qualificação de uma estratégia ou ação no serviço de saúde (DONABEDIAN, 2005; DONABEDIAN, 2003). Para qualificar um serviço de saúde, Donabedian (1990) utiliza-se da caracterização do que denomina os "sete pilares da qualidade": aceitabilidade, otimização, eficácia, efetividade, eficiência, equidade e legitimidade.

Atualmente, a avaliação da qualidade em saúde também se utiliza de conceitos relativos à gestão da qualidade a fim de definir as melhores padrões e normas de produção conforme os critérios técnicos disponibilizados (CASTANHEIRA, 2002). Segundo Nogueira (1994) e Novaes (1999), o principal foco na definição da qualidade é o cliente. Relativo à participação do cliente ou usuário, pode-se dizer que indiretamente está presente neste processo. Os clientes representam os atores fundamentais para os quais as ações avaliativas são direcionadas, sua atuação estará apresentada pelo valor positivo disponível nas relações dialéticas entre usuário e serviço de saúde (CASTANHEIRA, 2002).

Para Nemes et al. (2004), a avaliação da assistência em pessoas vivendo com HIV/Aids (PVHA), com foco nos parâmetros de qualidade, caracterizam-se por valorizar e evidenciar as ações que possibilitem o aumento da sobrevida e da qualidade de vida às PVHA, promovam a cidadania e o respeito à individualidade; o respeito e incentivo ao diálogo democrático com os usuários, assegurem acessibilidade dos alvos; coesão de atividades e valorização do trabalho em equipe; especifiquem as orientações na introdução medicamentosas para a melhora da adesão; norteiem os pacientes em relação a medidas de prevenção; autocuidado e vida reprodutiva; orientam parceiros; ofereçam ações direcionadas às mulheres, especialmente gestantes e recém-nascidos.

Assim, no Brasil, afim de se conhecer a necessidade, as características da assistência e avaliar a qualidade de serviços tão diversos, o Ministério da Saúde, por meio do 
Programa Nacional de DST e Aids (PNDST/Aids) estabeleceu parcerias com a equipe Qualiaids (formada por professores e pesquisadores) com a meta de criação de mecanismos de monitoramento e avaliação que contribuam para o aprimoramento da assistência às pessoas que vivem com

\section{METODOLOGIA}

O presente estudo refere-se a uma Revisão Interativa (RI) que mescla informações da literatura teórica e empírica, assim como incorpora uma variedade de proposições conceituais, análise metodológica, evidências ou revisão de teorias. As múltiplas proposições devem gerar uma visão panorâmica coesa e compreensível da teoria ou problema em análise (WHITTEMORE; KNAFL, 2005). A RI é utilizada no contexto da prática baseada em evidências (PBE) a qual é considerada um método de pesquisa que permite a análise ordenada e sistematizada de estudos importantes, que fornecerão a base para a tomada de decisões futuras e no aperfeiçoamento da prática clínica (BENEFIELD, 2003; MENDES, SILVEIRA, GALVÃO, 2008; ROMAN, FRIEDLANDER, 1998).
HIV/Aids (PVHA) atendidos no Sistema Único de Saúde (SUS) (NEMES et al., 2013).

Desse ao exposto, o nosso estudo analisa as evidências científicas relacionadas aos critérios de avaliação da atenção as pessoas que vivem com HIV/Aids.

Para a estruturação dessa revisão interativa, foram seguidos os passos propostos por Whittemore e Knafl (2005).

2.1 - FORMULAÇÃO DA HIPÓTESE E OBJETIVOS DO ESTUDO

Quais as evidências científicas relacionadas aos critérios de avaliação da assistência oferecidas em pessoas que vivem com HIV/Aids?

\section{2 - ESTABELECIMENTO DE} CRITÉRIOS E BUSCA

\section{SISTEMATIZADA DOS ARTIGOS}

Os descritores utilizados para a pesquisa dos estudos primários nas bases de dados foram: A AND B AND $C$ OR D AND E AND F; em que:

A - Quality Assurance, Health Care / Garantia da Qualidade dos 
Cuidados de Saúde; B - Health Services / Serviços de Saúde; C Acquired Immunodeficiency Syndrome I Síndrome de Imunodeficiência Adquirida; D - HIV / HIV; E - Process Assessment (Health Care) / Avaliação de Processos (Cuidados de Saúde); F Health Care Evaluation Mechanisms / Mecanismos de Avaliação da Assistência à Saúde

\section{3 - AVALIAÇÃO DOS DADOS}

Os estudos selecionados obedeceram aos critérios a seguir:

Os Critérios de inclusão: Estudos que avaliam a assistência ou 0 cuidado à pessoa que vive com HIV/Aids; Estudos publicados nos idiomas inglês, espanhol ou português. Os critérios de exclusão: Todos os artigos de revisão; artigos que não apresentem resumo; artigos que relatem trabalhos parciais ou não concluídos. A seguir o diagrama 1 sintetiza o processo de busca e seleção dos estudos incluídos nesta RI.

Diagrama 1 - Processo de busca e seleção dos estudos

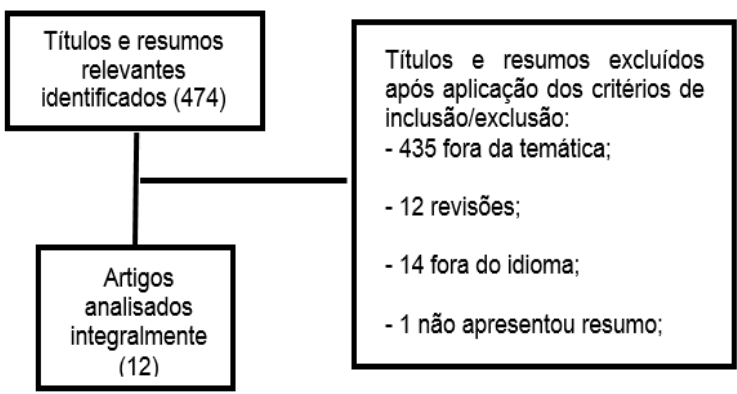

A Prática Baseada em Evidências nos permite, como requisito para implementação na prática profissional, a aplicação de um sistema de classificação de evidências hierárquicas do nível I a VII, conforme descrito por Melnyk; Fineout-Overholt, 2011, sendo o nível I de melhor evidência e o VII o nível menor evidência.

\section{4 - ANÁLISE DOS ESTUDOS} SELECIONADOS

A extração dos dados dos estudos primários presentes nessa RI foi realizada por dois revisores de modo independente. Para esse fim, foi utilizado o instrumento elaborado por Ursi (2005) adaptado e foi submetido a validação aparente e de conteúdo pelo autor e pelo segundo revisor.

\section{5 - APRESENTAÇÃO DOS} RESULTADOS

A apresentação dos resultados evidenciados na $\mathrm{RI}$ foi realizada na forma descritiva, a qual forneceu informações de cada estudo primário incluídos. 


\section{RESULTADOS E DISCUSSÃO}

Os artigos selecionados corresponderam a estudos publicados em periódicos internacionais, entre os anos de 1998 e 2015, sendo o idioma predominante o inglês $(91,66 \%)$ e o tipo de estudo mais utilizado o quantitativo transversal $(33,33 \%), \quad$ havendo al, 2012) e Box et al (2003) entre os achados. Apenas três estudos (25\%) empregaram o método misto, sendo o último um estudo brasileiro.

O quadro 1 sintetiza as principais características dos estudos incluídos nesta revisão.

$(16,66 \%)$ ensaios clínicos controlados randomizados (Finocchario-Kessler, et

Quadro 1 - Distribuição dos estudos incluídos segundo autor (ano), delineamento, nível de evidência, idioma e país de origem do estudo

\begin{tabular}{|c|c|c|c|c|}
\hline Autor (ano) & Delineamento & $\begin{array}{l}\text { Nível de } \\
\text { evidência }\end{array}$ & Idioma & $\begin{array}{l}\text { País de } \\
\text { Origem }\end{array}$ \\
\hline An et al. (2015) & Método misto & VI & Inglês & Tanzânia \\
\hline Keller et al. (2014) & Quantitativo transversal & VI & Inglês & EUA \\
\hline $\begin{array}{c}\text { Finocchario-Kessler, } \\
\text { et al. (2012) }\end{array}$ & $\begin{array}{l}\text { Ensaio clínico controlado } \\
\text { randomizado }\end{array}$ & II & Inglês & EUA \\
\hline $\begin{array}{l}\text { Alemayehu; Bushen; } \\
\text { Muluneh (2009) }\end{array}$ & Quantitativo transversal & VI & Inglês & Etiópia \\
\hline $\begin{array}{c}\text { Mapunjo; Urassa } \\
\text { (2007) }\end{array}$ & Quantitativo transversal & VI & Inglês & Tanzânia \\
\hline $\begin{array}{l}\text { Landman et al. } \\
(2006)\end{array}$ & Quantitativo transversal & VI & Inglês & Tanzânia \\
\hline $\begin{array}{l}\text { Davis-Michaud et al. } \\
\text { (2004) }\end{array}$ & Método misto & VI & Inglês & EUA \\
\hline $\begin{array}{l}\text { Chama, Audu; Kyari, } \\
\text { (2004) }\end{array}$ & Série de casos & VI & Inglês & Nigéria \\
\hline Box et al. (2003) & $\begin{array}{l}\text { Ensaio clínico controlado } \\
\text { randomizado }\end{array}$ & II & Inglês & EUA \\
\hline Mir et al. (2001) & Estudo populacional & VI & Inglês & Escócia \\
\hline Gomes et al. (1999) & Método misto & VI & Português & Brasil \\
\hline $\begin{array}{c}\text { Cunningham et al. } \\
\qquad(1998)\end{array}$ & Estudo de coorte & IV & Inglês & EUA \\
\hline
\end{tabular}


Os 12 estudos que avaliaram a atenção a PVHA em contextos diversos, ou seja, ampla variedade de provimento de recursos nas localidades; em sistemas públicos ou privados; os impasses e dificuldades técnicas para implantação ou execução da assistência em PVHA.

Os principais critérios de avaliação da a PVHA evidenciados nos artigos incluídos nesta $\mathrm{Rl}$ foram disponibilidade de recursos e infraestrutura (50\%); testagens para HIV e coinfecções (41,66\%); o aconselhamento (25\%) e acesso ou acessibilidade ao serviço de saúde $(16,66 \%)$. No entanto, critérios de avaliação como acolhimento, notificação de parceiro, cuidados da mulher e da criança (transmissão vertical), treinamentos ou capacitações da equipe de saúde e controle de registros tiveram pouca abordagem $(8,33 \%)$.

Dentre os 12 estudos incluídos nesta revisão, chamam a atenção para critérios de avaliação aplicados pelos estudos realizados por An et al. (2015), Keller et al (2014), Davis-Michaud et al. (2004), Chama, Audu; Kyari, (2004), Mir et al. (2001), Gomes et al. (1999) e Cunningham et al. (1998), pois resumem os parâmetros importantes de avaliação da assistência em PVHA.

An et al. (2015), utilizando-se de método misto, avalia a assistência em PVHA no contexto do pré-natal. São avaliados a infraestrutura para aconselhamento e testagens para HIV, disponibilidade de suprimentos e de equipe para a terapia antirretroviral. Este estudo indicou, como resultado, estruturas inadequadas para o cuidado pré-natal em PVHA, em especial, no parâmetro de disponibilidade de equipe e condições para a integração de aconselhamento e testagem para HIV

Para Walsh et al. (2010), a integração da testagem para HIV e aconselhamento adicionaram responsabilidades que aumentaram o tempo necessário para conduzir o aconselhamento, que por efeito aumentaram as filas. Kapologwe, Kabengula e Msuya (2011) descrevem que prestadores de saúde sentiam-se impotentes e sem envolvimento. O desestímulo apresentado deve-se, possivelmente, ao fato de que número reduzido de pessoal aumentou a carga de trabalho e, portanto, aumenta o cansaço dos funcionários.

Seguindo o modelo de estruturaprocesso-resultado proposto por Donabedian (2005), Keller et al. (2014) 
avalia a qualidade geral do cuidado de saúde com foco em medidas clínicas tradicionais tais como quantidade de consultas médicas e tempo de permanência no cuidado; testagem de HIV (carga viral e contagem de linfócitos TCD4) e triagem para coinfecções. O estudo conclui que, em geral as PVHA apresentaram indicadores de qualidade assistenciais satisfatórios, embora a triagem para comorbidade tenha apresentado baixa performance. Estudos realizados por Cohen et al (2011) e Bloch et al (2012) também apresentaram a conformidade de medidas específicas do cuidado do HIV e não conformidade para triagem de comorbidades.

A revisão sistemática de Catumbela et al. (2013) aponta para um conjunto de indicadores de qualidade a serem avaliados no cuidado clínico do paciente HIV/Aids, dentre eles estão a avaliação da carga viral e contagem de linfócitos TCD4 como também triagem para coinfecções, tais como tuberculose, hepatites B e C e câncer do colo uterino. Neste estudo, concluiuse que os esforços para identificar um conjunto de indicadores capaz de avaliar o cuidado clínico do HIV/Aids devem levar em consideração os aspectos da avaliação inicial, triagem, vacinação, terapia e monitoramento do tratamento.

Entendemos que resultados de conformidade são, em parte, adequados com o projeto programático avaliado, associado à coesão entre prestador da assistência e os pacientes. Contudo, deve-se propor aos prestadores de cuidados que tenham também foco na triagem de coinfecções, assim como em ações de vacinação.

Chama; Audu; Kyari (2004) visando contribuir com a redução da transmissão do HIV, avaliaram a assistência de saúde em PVHA utilizando-se dos parâmetros do aconselhamento e testagem para HIV entre gestante para a prevenção vertical. A intervenção aplicada pelos pesquisadores para prevenir a transmissão mãe para filho do HIV incluiu, além do aconselhamento e testagem voluntários (VCT), a oferta e administração de drogas antirretrovirais, modificação na prática obstétrica e alternativas de alimentação dos bebês de mães HIV.

A revisão sistemática produzida por Ambia e Mandala (2016) ao avaliarem estratégias de intervenção para melhorar a prevenção da transmissão vertical mostram que 0 
envolvimento dos parceiros homens das mães HIV como também o envio de mensagens via dispositivo móvel podem aumentar o número de diagnósticos recentes de recémnascidos de HIV. Diante disto, entendemos que o comprometimento de parceiros na terapia e a o uso de tecnologias apresentam-se como possíveis estratégia viáveis para 0 aumento e a manutenção dos usuários nos cuidados assistenciais.

Mir et al. (2001) avalia o processo de notificação dos parceiros de pacientes HIV/Aids. Observou-se neste estudo que houve $23 \%$ de notificações de parceiros que, quando testados para HIV, resultaram em novos diagnósticos.

A notificação de parceiro no Reino Unido é uma estratégia de controle das infecções sexualmente transmissíveis (IST) bem definida e defendida pelo Estado e pela Associação Britânica do HIV (BHIVA) (BRITISH HIV ASSOCIATION, 2014; REINO UNIDO, 1992). Outros sim, as diretrizes da BHIVA defendem 0 tratamento antirretroviral antes de ocorrer os danos imunológicos (BRITISH HIV ASSOCIATION, 2014; BRITISH HIV ASSOCIATION, 2000).
No Brasil, a notificação de parceiros está apresentada dentro do processo de ação de profissionais enfermeiros, psicólogos e assistente sociais no ato de aconselhamento das PVHA e em conformidade com os princípios e diretrizes do PN-DST/Aids (NEMES, 2013).

O parâmetro apresentado pelo estudo de Mir et al. (2001) é importante ponto de avaliação, pois trata-se de uma estratégia que visa ampliar o número de diagnóstico, garantir 0 acesso ao tratamento

Davis-Michaud et al. (2004) e Gomes et al. (1999) avaliam a qualidade da assistência em PVHA frente a perspectiva do paciente. Nestes estudos são avaliados os atributos de acesso, utilização, preferências dos clientes e estrutura física. Destaca-se no estudo 11 a avalição da disponibilidade de profissionais de saúde na assistência à PVHA.

Estes estudos, na medida em que buscam avaliar a qualidade do centro de saúde ao paciente HIV/Aids, abordando a perspectiva do cliente/paciente, vão além de avaliar atributos de organização geral e disponibilidade de recurso; avaliam o que para Nogueira (1994) e Novaes 
(1999) é o principal na relação prestador-usuário: o foco no cliente. Estendendo esta relação para serviçousuário-comunidade, Nemes et al. (2013a) atribui dois pontos relevantes:

A participação da comunidade no direcionamento da assistência, ou seja, em seu gerenciamento e organização e - acesso dos usuários a espaços sigilosos para manifestação de queixas sem prejuízos de qualquer natureza.

Cunningham et al. (1998) avaliam a qualidade da assistencial em PVHA fazendo uso dos seguintes critérios: acessibilidade, disponibilidade, conveniência e acesso a especialistas. Ademais, também foram coletados dados sobre o uso de antirretrovirais e medicação profilática para Pneumocystis pneumonia (PCP). Cunningham et al. (1998) avaliaram também o efeito do acesso aos cuidados médicos na qualidade de vida de pessoas com HIV/Aids a partir dos seguintes parâmetros: acessibilidade, disponibilidade, conveniência no cuidado médico e contagem de linfócitos TCD4. Neste estudo os autores concluíram que o acesso ao cuidado não melhorou 0 resultado para a média dos participantes, sendo observada melhora em subgrupos que estavam no terço médio da saúde física no momento da inscrição.

Atributos clínicos convencionais de avaliação como contagem de linfócitos TCD4 e carga viral do HIV não devem ser realizados de modo dissociados, isto é, devem estar ligados a avaliação de acessibilidade, gerenciamento técnico, disponibilidade de recursos e de medicamentos antirretrovirais, assim como devem priorizar ações de combate e prevenção de nova infecções visando o bem-estar social e a melhor qualidade de vida do paciente.

O acesso aos cuidados médicos para PVHA é amplamente reconhecido como importante, mas ainda problemático. Os pacientes pobres recebem atendimento em centros de saúde públicos e neste cenário tendem a ser mais vulneráveis a encontrar barreiras, independentemente da doença. Frente a isto, Markson et al (1997) relatam que devem ser estabelecidos programas de melhoria de acesso aos cuidados visando aqueles pacientes mais propensos a usufruírem os benefícios.

Neste sentido, Nemes et al. (2009) descreveram em seus estudos que o PN-DST/Aids, desde o final dos anos 90 , recomenda que a adesão seja 
item prioritário nas ações assistenciais dos serviços do SUS que atendem às PVHA.

Ententemos, portanto, que o acesso está associado à adesão a terapia antirretroviral e esta pode ser entendida como um preditivo positivo de qualidade de vida, visto que, de acordo com Silva et al (2014) melhora a imunidade, controla a carga viral e retarda a progressão da doença.

Do nosso ponto de vista, em que pese os benefícios, muitas pessoas que vivem com 0 HIV/Aids têm dificuldades de adesão ao tratamento, relacionadas não só aos reflexos clínicos da terapia, como também pela dificuldade de acesso ao serviço e, com efeito, de acesso às drogas antirretrovirais.

Quando se buscam evidências para a avaliação da assistência em pessoas que vivem com HIV/Aids, deve-se apontar para a perspectiva de programas ou ações que sejam positivas para o cliente ou seja, que denotem boa qualidade.

Diante do perfil anteriormente traçado por Castanheira (2002) e Nemes (2004), buscou-se evidências na literatura científica que convergissem com as categorias avaliativas propostas por Nemes et al. (2013a), descritas anteriormente. Assim, neste estudo, as categorias "organizacional", "gerenciamento técnico" e "disponibilidade de recursos", são desdobradas nas seguintes categorias: "assistencial" (organizacional e disponibilidade de recursos) e "gerenciamento técnico", afim de obter melhor compreensão.

Considerando, portanto, as categorias propostas acima, a análise dos artigos desta revisão permitiu a inclusão destes 12 estudos em apenas uma categoria em comum: a assistencial.

Após a análise dos estudos apresentados nesta revisão integrativa (RI) e fundamentado na literatura científica, a tabela 1 apresenta a síntese dos critérios de avaliação da assistência em PVHA evidenciados em cada estudo.

As limitações desta RI foi o fato de que ao longo do período pesquisado não evidenciamos critérios de gerenciamento técnicos como parâmetro principal de avaliação. Limitando-nos a evidenciar os atributos positivos presente em cada estudo e não a definirmos qual conjunto de critérios avaliativos em assistência ao PVHA mais adequado. 
Tabela 1 - Síntese dos critérios de avaliação

\begin{tabular}{|c|c|}
\hline Autores (ano) & Critérios de Avaliação \\
\hline An et al. (2015) & $\begin{array}{l}\text { Aconselhamento; testagens HIV; infraestrutura; } \\
\text { disponibilidade de suprimentos; terapia antirretroviral. }\end{array}$ \\
\hline Keller et al. (2014) & $\begin{array}{l}\text { Número de consultas médicas; tempo de permanência } \\
\text { na terapia; testagens HIV e triagem de coinfecções. }\end{array}$ \\
\hline $\begin{array}{l}\text { Finocchario-Kessler, et al. } \\
\qquad(2012)\end{array}$ & $\begin{array}{l}\text { Aderência; conhecimento sobres a terapia } \\
\text { antirretroviral; satisfação sobre o aconselhamento. }\end{array}$ \\
\hline $\begin{array}{l}\text { Alemayehu; Bushen; Muluneh } \\
\text { (2009) }\end{array}$ & $\begin{array}{l}\text { Treinamento da equipe de saúde; infraestrutura; } \\
\text { disponibilidade da terapia antirretroviral; disponibilidade } \\
\text { de profilaxia para coinfecções e satisfação do paciente. }\end{array}$ \\
\hline Mapunjo; Urassa (2007) & $\begin{array}{l}\text { Disponibilidade de equipamentos e antirretrovirais; } \\
\text { conhecimentos das diretrizes sobre a terapia } \\
\text { antirretroviral; recursos humanos e treinamento; } \\
\text { controle de registros da terapia antirretroviral. }\end{array}$ \\
\hline Landman et al. (2006) & $\begin{array}{l}\text { Disponibilidade de recursos humanos e treinamento; } \\
\text { condições das instalações clinicas e laboratoriais para } \\
\text { prover a terapia antirretroviral. }\end{array}$ \\
\hline Davis-Michaud et al. (2004) & $\begin{array}{l}\text { Acesso; utilização do serviço de saúde; infraestrutura; } \\
\text { preferências do paciente; relação com a equipe de } \\
\text { saúde. }\end{array}$ \\
\hline Chama, Audu; Kyari, (2004) & $\begin{array}{l}\text { Intervenções para a redução da transmissão vertical; } \\
\text { aconselhamento e testagens HIV e disponibilidade de } \\
\text { antirretrovirais. }\end{array}$ \\
\hline Box et al. (2003) & $\begin{array}{l}\text { Acesso; testagens HIV e disponibilidade de profilaxia } \\
\text { para coinfecções. }\end{array}$ \\
\hline Mir et al. (2001) & Notificação de parceiros. \\
\hline Gomes et al. (1999) & $\begin{array}{l}\text { Acesso; características do usuário; acolhimento; } \\
\text { disponibilidade de profissionais de saúde e percepção } \\
\text { geral do atendimento. }\end{array}$ \\
\hline Cunningham et al. (1998) & $\begin{array}{l}\text { Acessibilidade a especialistas; disponibilidade e } \\
\text { conveniência da assistência; Contagem de linfócitos } \\
\text { TCD4; adesão ao antirretroviral e profilaxia para }_{\text {Pneumocystis pneumonia (PCP). }}\end{array}$ \\
\hline
\end{tabular}

\section{CONSIDERAÇÕES FINAIS}


Os estudos apontaram evidências de critério de avaliação baseados no acesso, aconselhamento, na disponibilidade de recurso, na prevenção da transmissão vertical, e na relação usuário-serviço de saúde, além dos convencionais critérios clínicos de avaliação de células TCD4, carga viral e triagem de coinfecções.

Contemplando um dos escopos da Prática Baseada em Evidências (PBE), cuja aplicação é multiprofissional, a implementação de resultados de pesquisa contribui para a reflexão e a mudança da prática assistencial dos profissionais de saúde. Para isso, como desafio inicial, deve-se compreender que 0 interesse e 0 preparo dos profissionais frente às pesquisas são importantes.

Assim, considerando aprimorar a prática assistencial a fim de assegurar o bem-estar das pessoas que vivem com HIV/Aids, sugerimos elaboração de um guia de diretrizes, fundamentado nos critérios de avaliação apresentados tabela - 1 desta revisão, que possa ser aplicado nos serviços de saúde em seus diferentes níveis da atenção e permita assegurar a adequada qualidade na assistência em PVHA. Ademais, recomenda-se a realização de novos estudos de rigoroso delineamento metodológico a fim de que possam surgir reflexões quanto a possível elaboração de diretrizes assistencial universal às PVHA. Fazem-se também necessários estudos que destaquem as condições dos registros, atualização dos profissionais, planejamento e coordenação do trabalho, visto que são essas ações norteiam os profissionais de saúde em suas condutas e impactam a percepção do cliente.

\section{REFERÊNCIAS}

AGUILAR, M.J; ANDER-EGG, E. Avaliação de serviços e programas sociais. Petrópolis: Vozes, 1994.

AMBIA, J; MANDALA, J. A systematic review of interventions to improve prevention of mother-to-child HIV transmission service delivery and promote retention. Journal of the International AIDS Society, v. 19, n. 1, 2016, p. 203-209. 
$\mathrm{BLOCH}$, M. et al. Adherence to HIV treatment guidelines for comorbid disease assessment and initiation of antirretroviral therapy. Journal of Acquired Immune Deficiency Syndromes, v. 59, n. 5, 2012, p. 478-488.

BRASIL. Ministério da Saúde. Departamento Nacional de DST, Aids e Hepatites Virais. Brasília $\quad$ (DF), 2016.2 Disponível em $<$ http://www.aids.gov.br/tipo_endereco/servico-de-assistencia-especializada-emhivaids > . Acessado em 22 de maio de 2016.

BRITISH HIV ASSOCIATION. British HIV Association guidelines for the treatment of HIV-1-positive adults with antiretroviral therapy 2012. London: HIV Medicine, v. 15 (Suppl. 1), 2014, p.1-85.

CASTANHEIRA, E.R.L. Avaliação da assistência ambulatorial a pessoas vivendo com HIV/aids em serviços públicos no estado de São Paulos: relações entre qualidade e organização do processo de trabalho. Tese de doutorado. São Paulo: Faculdade de Medicina de USP, 2002, 161 p.

CATUMBELA et al. Definition of a core ser of quality incdicators for the assessment of HIV/AIDS clinical care: a systematic review. BMC Health Services Research, v. 13, 2013, p.236.

COHEN, S.M; VAN HANDEL, M.M; BRANSON, B.M. et al. Vital Signs: HIV prevention througth care and treatment - United States. v. 60, n. 47, 2011, p.1618-1623,. Disponível em <https://www.cdc.gov/mmwr/preview/mmwrhtml/mm6047a4.htm>. Acessado em: 20/10/2016.

DONABEDIAN, A. Evaluating the quality of medical care. 1966. The Milbank Quarterly, v. 83, n. 4, 2005, p.691-729.

DONABEDIAN, A. An introduction to quality assurance in health care. New York: Oxford University Press, 2003, 240 p.

DONABEDIAN, A. The seven pillars quality. Archives of Pathology \& Laboratory Medicine Journal, v. 114, n. 11, 1990, p.1115-1118.

GODOY, M.R; SOUSA, T.R.V; SILVA, E.N. The impact of socio-economic status on incidence of AIDS cases in Brazilian cities: a study in panel data. Londrina: Economia \& Região, v.1, n. 1, jan-jul./2013, p. 4-25.

KAPOLOGWE, N.A; KABENGULA, J.S; MSUYA, S.E. Perceived barriers and attitudes of health care providers towards Provider-Initiated HIV Testing and Counseling in Mbeya region, southern highland zone of Tanzania. The Pan African Medical Journal. 2011, v. 8, p. 17

MARKSON, L.E et al. Clinic sevices for persons with AIDS experience in a higthprevalence state. Journal of General Internal Medicine, v.12, n. 3, 1997, p.141. 
MELCHIOR, R et al. Avaliação da estrutura organizacional da assistência ambulatorial em HIV/Aids no Brasil. Revista de Saúde Pública, v. 40, n. 1, 2006, p.143-151.

MENDES, K.D.S; SILVEIRA, R.C.C.P; GALVÃO, C.M. Revisão interativa: métodos de pesquisa para a incorporação de evidências na saúde e na enfermagem. Texto Contexto Enfermagem, v.17, n. 4, out-dez, 2008, p.758-764.

MELNYK, B. M.; FINEOUT-OVERHOLT, E. Making the case for evidence-based practice. In: MELNIK, B. M.; FINEOUT-OVERHOLT, E. Evidence-based practice in nursing and healthcare. A guide to best practice. 2a ${ }^{\underline{a}}$ Ed. Philadelphia: Lippincott Williams \& Wilkins, 2011, 487 p.

NEMES et al. Qualiaids: avaliação e monitoramento da qualidade e recomendações de boas práticas da assistência ambulatorial em aids no SUS. Caderno Qualiaids. 2013, 110 p.

NEMES, M.I.B et al. Adesão ao tratamento, acesso e qualidade da assistência em aids no Brasil. Revista da Associação Medica Brasileira. v. 55, n. 2, 2009, p.207212.

NEMES, M.I.B et al. Avaliação da qualidade da assistência no programa de AIDS: questões para a investigação em serviços de saúde no Brasil. Caderno Saúde Pública, Rio de Janeiro, v. 20, Sup 2, 2004, p.S310-S321.

NOGUEIRA, R.P. Perspectivas da qualidade em saúde. Rio de Janeiro-RJ: Qualitymark. 1994, 155 p.

NOVAES, H.M.D. Mortalidade neonatal e avaliação da qualidade de atenção ao parto e ao recém-nascido no município de São Paulo. Tese de livre docência. São Paulo: Faculdade de Medicina de USP, 1999.

REINO UNIDO. Department of Health. Guidance on partner notification in HIV infection. London. PL/CO, (92)5, Appendix 3, Dec 1992.

REINO UNIDO. Health Education Authority Council. A handbook on contacttracing in sexually transmitted diseases. London: Health Education Council, 1980.

ROMAN, A.R; FRIEDLANDER, M.R. Revisão interativa de pesquisa aplicada à enfermagem. Cogitare Enfermagem, v. 3, n. 2, jul-dez. 1998, p.109-112.

SILVA, A.C.O et al. Qualidade de vida, características clínicas e adesão ao tratamento de pessoas vivendo com HIV/AIDS. Revista Latino-Americana de Enfermagem, v.22, n.6, nov.-dez. 2014, p.994-1000.

UNAIDS Joint United Nations Programme on HIV/Aids. AIDS by the numbers. 2016. Geneve. Word Heath Organization Disponível em:<http://www.unaids.org/en/resources/documents/2016/AIDS-by-the-numbers>. Acessado em: 24/11/2016. 
UNAIDS Joint United Nations Programme on HIV/Aids. Global Report Up Date 2016. 2016a. Geneve: Word Heath Organization. Disponível em: http://www.unaids.org/sites/default/files/media_asset/global-AIDS-update2016_en.pdf. Acessado em 24/11/2016.

UNAIDS. UNAIDS do Brasil. A ONU e a resposta à AIDS no Brasil. 2012. Disponível em: $\quad$ <http://unaids.org.br/wp-content/uploads/2016/03/A-ONU-e-a-respostaPORTUGUÊS.pdf>. Acessado em: 15/10/2016.

URSI, E. S. Prevenção de lesão de pele no perioperatório: versão integrativa da literatura. 2005.128 f. Dissertação (Mestrado) - Escola de Enfermagem de Ribeirão Preto, Ribeirão Preto: Universidade de São Paulo, 2005.

WALSH, A et al. Task sharing in Zambia: HIV service scale-up compounds the human resource crisis. BMC Health Services Research, v. 10, 2010, p.272.

WORLD HEALTH ORGANIZATION (WHO). HIV/AIDS and other sexually transmitted infections. Disponível em: <http://www.who.int/ith/diseases/hivaids/en/>. Acesso em: 19 mai 2016.

WHITTENMORE, R; KNAFL, K. The interativa review: updated methodology. Blackwell Publishing Ltd. Journal of Advanced Nursing, v. 52, n. 5, 2005; p.546-553. 